\title{
Reduction of Titanium Dioxide to Metallic Titanium by Thermal Decomposition via Titanium Disulfide
}

\author{
Ichiro Seki* \\ National Institute of Technology, Tsuyama College, Tsuyama 708-8509, Japan
}

We have earlier reported the synthesis of metallic titanium ingot by thermal decomposition process using titanium nitride (TiN) as the intermediate material, with a significant low decomposition temperature of approximately $3500 \mathrm{~K}$. The present study reports the manufacturing process of metallic titanium ingot via thermal decomposition using titanium sulfides $\left(\mathrm{TiS}_{\mathrm{X}}\right)$ as intermediate materials: decomposition temperature of one of the titanium sulfides, TiS, is approximately $4000 \mathrm{~K}$. The thermal decomposition was performed by an arc-flame in a conventional arc melting equipment. The reduced product exhibiting a lustrous surface is analyzed by XRD, and is matched with diffraction pattern of metallic titanium. [doi:10.2320/matertrans.MT-MAW2021002]

(Received May 12, 2021; Accepted July 21, 2021; Published September 25, 2021)

Keywords: thermodynamics, thermolysis, titanium sulfide, Gibbs free energy

\section{Introduction}

The Kroll method is used in industries for the production of sponge titanium with high purity, ${ }^{1)}$ wherein raw titanium dioxide $\left(\mathrm{TiO}_{2}\right)$ ore, an upgraded ilmenite, is converted to titanium tetrachloride $\left(\mathrm{TiCl}_{4}\right)$ in the presence of coke and chlorine gas, followed by a carbochlorination reaction. Then, metallic magnesium $(\mathrm{Mg})$ is used to reduce the $\mathrm{TiCl}_{4}$ intermediate. The metallic magnesium and chlorine gas are electrochemically recycled from the reduction product of magnesium chloride $\left(\mathrm{MgCl}_{2}\right)$ via electrolysis, thus requiring a large amount of electricity. Moreover, the reaction chamber requires a prolonged cooling time, since the reduction reaction is highly exothermic in nature. Then, $\mathrm{Mg}$ and $\mathrm{MgCl}_{2}$ residues are removed via a slow vacuum distillation process, and sponge-like metallic titanium is formed. Finally, the produced sponge titanium is consolidated into the shape of an electrode, and this consumable electrode is melted in an arc melting furnace to form titanium ingot. The conventional process of manufacturing metallic titanium requires complex processes as well as high manufacturing costs.

Owing to the complex industrial manufacturing process, several researchers have focused on exploring novel processing methods for the production of metallic titanium, and recent researches are summarized by Suzuki et al. ${ }^{2)}$ Some researchers have reported the synthesis of metallic titanium particles employing an electrochemical method, with molten calcium chloride or molten salt as the medium, ${ }^{3-6)}$ whereas others have also proposed producing metallic titanium particles through magnesiothermic and calciothermic reductions using molten salts. ${ }^{7,8)}$ In our previous study, we reported a thermolysis (thermal decomposition) method for the production of titanium ingots. ${ }^{9)}$ Titanium nitride (TiN) with a low decomposition temperature was used as the intermediate material. TiN was easily produced from $\mathrm{TiO}_{2}$ in a furnace, under the carbon potential-controlled nitrogen gas atmosphere, ${ }^{10)}$ and also decomposed to metallic titanium ingot instantly by arc-melting, which can easily perform the decomposition temperature immediately. The thermolysis

*Corresponding author, E-mail: seki@tsuyama-ct.ac.jp method for production of metallic titanium ingots is a reasonable technique for high-speed and mass production. However, the purity issue of the produced titanium ingots inhibits the replacement of conventional process with the proposed thermolysis method proposed.

The present study also focuses on the thermal decomposition method and uses titanium-based intermediate materials. Sulfur is easily delivered from everywhere and is economically available well. Titanium sulfide $\left(\mathrm{TiS}_{\mathrm{X}}\right)$ is one of the titanium-based intermediates having low decomposition temperature approximately about $4000 \mathrm{~K}$, which is similar to that of $\operatorname{TiN}(3500 \mathrm{~K}) .{ }^{11)} \mathrm{TiS}_{\mathrm{X}}$ is treated as the intermediate material for titanium production, and is already electrochemically performed in molten salt. ${ }^{12,13)}$ Moreover, TiSx can be formed from $\mathrm{TiO}_{2}$ by sulfurization in carbon disulfide gas $\left(\mathrm{CS}_{2}\right)$ atmosphere, ${ }^{14,15)}$ and it can also be directly formed from TiN in sulfur gas atmosphere. ${ }^{16)}$ The thermodynamic calculations suggest that it is also possible to form TiSx by a carbo-sulfurization reaction of $\mathrm{TiO}_{2} .{ }^{11)}$ Hence, the present work proposes one of processing methods for the production of metallic titanium ingot from $\mathrm{TiO}_{2}$ powder. It can be anticipated that this processing technique, based on the chemical conversion of $\mathrm{TiO}_{2}$ to titanium sulfides in sulfur gas atmosphere and its subsequent thermal decomposition to metallic titanium will be an efficient and cost-effective processing method.

\section{Theoretical Background}

Ilmenite $\left(\mathrm{FeO} \cdot \mathrm{TiO}_{2}\right)$, which is a complex titanium-iron oxide ore, is industrially used for the manufacture of metallic titanium, after being upgraded to highly purified $\mathrm{TiO}_{2}$. The bonding strength of titanium and oxygen is significantly high as compared to its bonding with other elements. Figure 1 shows the differences in Gibbs free energy of formation of Ti oxides, nitride, sulfide and chloride by $1 \mathrm{~mol}$ gas in the temperature range of $1000-4000 \mathrm{~K}$, found from the thermodynamic data. ${ }^{11)}$ Here, $\mathrm{TiO}_{2}$ is simply regarded as up-graded ilmenite, $\mathrm{TiCl}_{4}$ is commonly used in the industrial production process as an intermediate material to manufacture metallic titanium, and $\mathrm{TiN}$ is used as the intermediate 


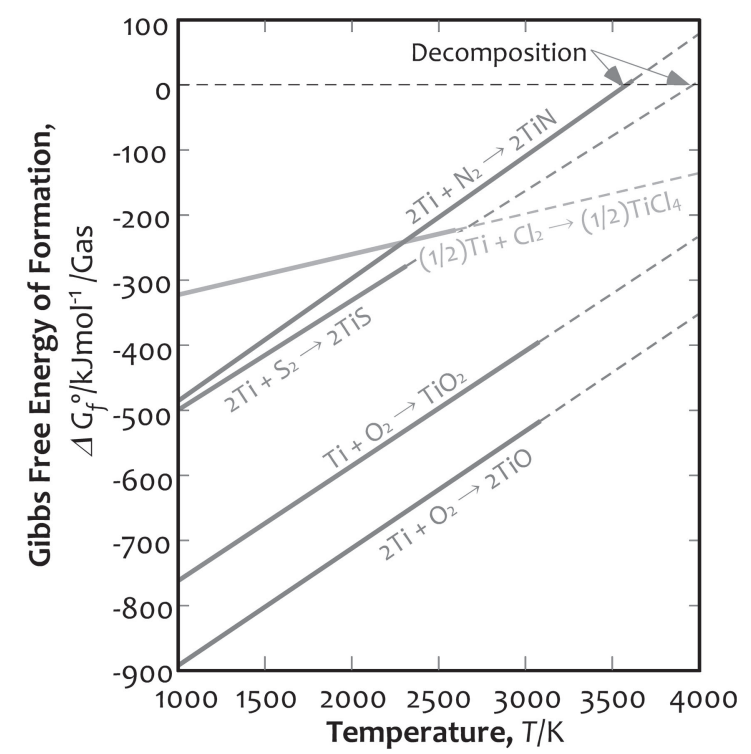

Fig. 1 Variation in the Gibbs free energies of formations for the titaniumbased compounds.

material used for the production of metallic titanium ingots using a thermal decomposition method, as shown in our previous report. ${ }^{9)}$ The thermolysis temperature of titanium sulfide (TiS) as one of reference materials of the titanium sulfides is also anticipated from the thermodynamic data (Thermodynamic data of $\mathrm{TiS}_{2}$ for high temperature region is not reported). $\mathrm{TiO}_{2}$ and titanium oxide (TiO) are typically highly stable in the wide temperature range, as demonstrated by their large negative values (Fig. 1) of Gibbs free energies of formation. $\mathrm{TiCl}_{4}$ also has a small negative Gibbs free energy of formation at lower temperature, i.e., $1000 \mathrm{~K}$, but it becomes similar to that of the titanium oxides at approximately $4000 \mathrm{~K}$. In contrast, TiN has a small negative Gibbs free energy of formation in a wide temperature range compared to that of titanium oxides, implying that $\mathrm{TiN}$ is easily reduced to metallic titanium as compared to titanium oxides. Moreover, the positive value of Gibbs free energy of formation of $\mathrm{TiN}$ at approximately $3500 \mathrm{~K}$ indicates its instability above this temperature as it thermolytically separates to form metallic titanium and nitrogen gas. Owing to the small negative Gibbs free energy of formation in a wide temperature range, TiS is also expected to thermolytically separate into metallic titanium and sulfur gas. ${ }^{11)}$ Generally, the thermal decomposition does not depend on the surface reaction. It is not accompanied by a reducing agent such as $\mathrm{Mg}$, and hence, it may expect a faster reaction in the present study.

For the application of this manufacturing process, it is necessary to produce the titanium sulfides as the intermediate materials. The formations of the titanium sulfides are also observed from the Gibbs free energy changes of both the direct and indirect reactions, as shown in Fig. 2. Here, indirect reaction implies combustion with carbon, also known as the carbothermic reaction. As indicated in the Fig. 2, the value of Gibbs free energy of formation is positive for the direct reaction, $\mathrm{TiO}_{2}+\mathrm{S}_{2} \rightarrow \mathrm{TiS}_{2}+\mathrm{O}_{2}$, which implies that the formation of $\mathrm{TiS}_{2}$ is not possible. However, the negative value of Gibbs free energy of formation for the indirect

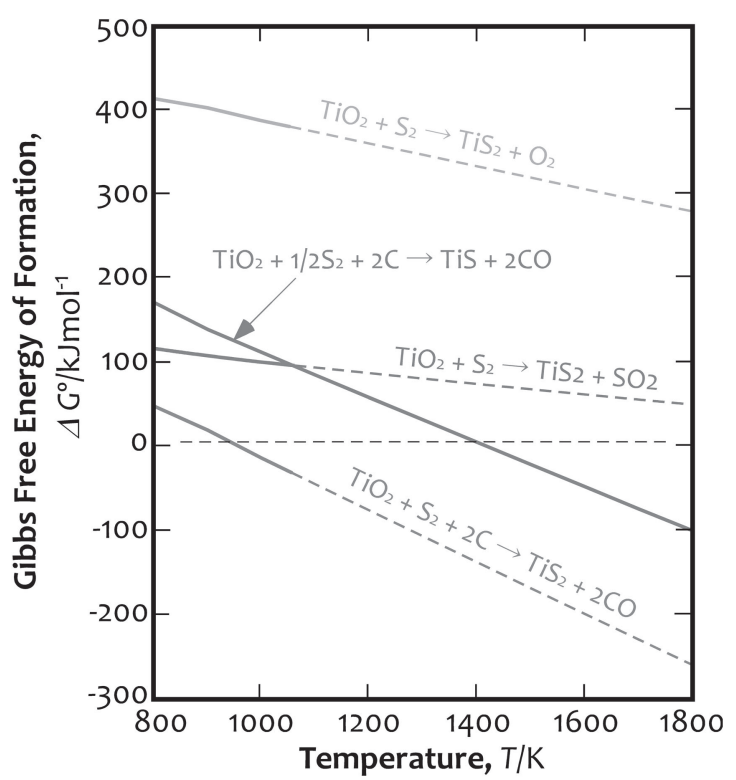

Fig. 2 Variation in the Gibbs free energies of formation of $\mathrm{TiS}_{2}$ from $\mathrm{TiO}_{2}$ by direct and carbothermic reactions.

reaction, i.e., $\mathrm{TiO}_{2}+\mathrm{S}_{2} \quad$ (or $\left.1 / 2 \mathrm{~S}_{2}\right)+2 \mathrm{C} \rightarrow \mathrm{TiS}_{2} \quad$ (or TiS) $+2 \mathrm{CO}$, suggest the formation of $\mathrm{TiS}_{\mathrm{X}}$ through this process. Here, the titanium oxides undergo multiple phase transformations to form $\mathrm{Ti}_{4} \mathrm{O}_{7}, \mathrm{Ti}_{3} \mathrm{O}_{5}$, and $\mathrm{TiO}$ with different degrees of oxidation. The titanium sulfides also form phases with different degrees of sulfurization. Hence, it is necessary to discuss these phases with different degrees of oxidation and sulfurization. A thermodynamic study using potential diagrams is described in the result section to obtain the possibility of thermal decomposition of titanium disulfides to metallic titanium.

\section{Experimental Procedure}

Anatase-type titanium dioxide $\left(\mathrm{TiO}_{2}\right)$ powder of reagent grade (Wako Pure Chemical Industries, Ltd.) was used in the present study. The reported values of thermodynamic properties ${ }^{11)}$ indicate that the anatase-type $\mathrm{TiO}_{2}$ decomposes easily than the rutile type. Hence, anatase-type $\mathrm{TiO}_{2}$ was preferred over rutile $\mathrm{TiO}_{2}$, anticipating a higher reactivity. $\mathrm{TiO}_{2}$ powder was mixed with regent grade graphite powder (Japan Pure Chemical Co., Ltd.), and placed in an alumina reaction boat. The reaction boat was covered with a hemisected carbon crucible and inserted into a quartz tube, as shown in Fig. 3(a). The reaction boat was also connected to a quartz test-tube containing a regent grade sulfur powder (Kishida Chemical Co., Ltd.). The mixture of $\mathrm{TiO}_{2}$ and graphite powder was heated to $1473 \mathrm{~K}$ for $7.2 \mathrm{ks}(2 \mathrm{~h})$ to $21.6 \mathrm{ks}(6 \mathrm{~h})$ inside a horizontal vacuum furnace in an argongas atmosphere maintained at an atmospheric pressure of $1 \mathrm{~atm}$. The sulfur powder was also heated from 373 to $773 \mathrm{~K}$ stepwise to supply sulfur gas to the mixture of anatase and graphite. The mixture was then rapidly cooled down to room temperature $(300 \mathrm{~K})$ under an argon-gas flow with $0.17 \mathrm{ml} / \mathrm{s}$ $(10 \mathrm{ml} / \mathrm{min})$. The synthesized product was then analyzed using an X-ray diffractometer (XRD) system (MiniFlex II, Rigaku Co.). The outer surface of the product before and after the synthesis is shown in Fig. 3(b). 

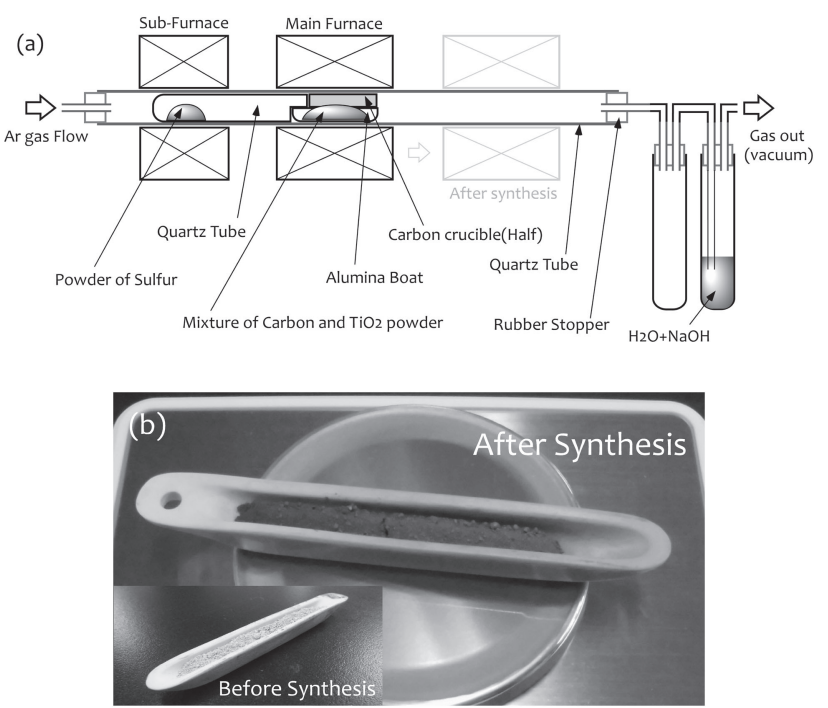

Fig. 3 (a) Schematic of the experimental equipment for the production of $\mathrm{TiS}_{2}$ from the mixture of regent $\mathrm{TiO}_{2}$ and graphite powders, and (b) photograph of the mixture before and after the synthesis.

For the conversion of titanium sulfide to metallic titanium, the synthesized mixture of titanium sulfide powder was inserted into a die with an inner diameter of $20 \mathrm{~mm}$ and compressed using an oil-hydraulic cylinder. The compressed mixture of titanium sulfide powder was then heated to 3000 $4000 \mathrm{~K}$ using a commercial arc melting furnace equipment (DIAVAC Ltd.) under a decompressed argon atmosphere. Here, the decomposition temperature of $3000-4000 \mathrm{~K}$ is difficult to perform by a common electric resistance furnace. Melting temperature of crucible made by alumina is about $2300 \mathrm{~K}$ and even that of calcia is under $3000 \mathrm{~K}$. Moreover, that temperature is different reacting with the elements of the melts, and its behavior becomes conspicuous depending on combinations of elements. In contrast, the arc-melting technique is easily performed that temperature, and it is unnecessary to consider material of crucibles and its complex combinations. Therefore, the arc-melting technique is selected in the present study. The compressed mixture was subjected to the arc melting several times, with the reaction chamber being cleaned after each step due to the staining caused by dew of sulfur during the process. The resulting products were analyzed using XRD.

\section{Results}

\subsection{Synthesis of titanium sulfides produced by the carbothermic reaction}

Figure 4(a) shows the XRD pattern of the mixture of anatase and graphite powders. The obtained XRD results matches with the standard diffraction patterns of Anatase (PDF \#01-075-1537, International Centre for Diffraction Data) and graphite (PDF \#01-071-3739, shows in the diffraction pattern of sp19), and was marked in the pattern of the obtained mixture. The diffraction pattern of the mixture obtained after it was heated to $1373 \mathrm{~K}$ was matched with the standard data of rutile (PDF \#01-071-3739) and graphite (PDF \#01-071-3739), as shown in Fig. 4(b). As reported by Kubo and Shinriki, ${ }^{17)}$ the rutile phase is stable above $1473 \mathrm{~K}$, and the anatase $\mathrm{TiO}_{2}$ is transformed to rutile $\mathrm{TiO}_{2}$ at around this temperature. The results shown in Fig. 4(a), (b) are traced well their conclusion. Hence, the reaction process in the present study can be roughly interpreted as the reduction of rutile type titanium oxide to titanium sulfides.

Figure 5(a)-(c) shows the XRD patterns of the heat-treated mixture at $1473 \mathrm{~K}$ for $7.2 \mathrm{ks}(2 \mathrm{~h}), 14.4 \mathrm{ks}(4 \mathrm{~h})$ and $21.6 \mathrm{ks}$ $(6 \mathrm{~h})$, respectively. The diffraction patterns presented in Fig. 5(a) match with the standard data of titanium sulfide $\left(\mathrm{Ti}_{1.19} \mathrm{~S}_{2}\right.$, PDF \#00-041-0930), and the presence of stoichiometric titanium disulfide $\left(\mathrm{TiS}_{2}\right.$, PDF \#03-065-3372) is clearly observed in the XRD patterns (Fig. 5(b) and (c)) of the mixture heat-treated for more than $14.4 \mathrm{ks}$. Here, $\mathrm{Ti}_{1.19} \mathrm{~S}_{2}$ is one of non-stoichiometric compounds of $\mathrm{TiS}_{2}$, and its crystallographic and thermodynamic relations are reported. ${ }^{18,19)}$ The titanium-rich non-stoichiometric compound, $\mathrm{Ti}_{1.19} \mathrm{~S}_{2}$, seems to be prepared by a little lower sulfur partial pressure than the production of $\mathrm{TiS}_{2}$. However, it is interpreted that the presented partial pressure of sulfur is enough for expulsion of oxygen from the $\mathrm{TiO}_{2}$ (convert from $\mathrm{TiO}_{2}$ to $\mathrm{TiS}_{\mathrm{x}}$ ). Although the rutile phase of titanium dioxides (Rutile, PDF \#01-071-0650 and PDF \#01-071-4809) are seen in the mixture heated for $7.2 \mathrm{ks}$, they seem to have disappeared from the mixture that was heat-treated for more than $14.4 \mathrm{ks}$. Graphite (PDF \#01-071-3739) is also slightly seen in all the mixtures. Figure 6(a) and (b) shows the diagrams for oxygen partial pressure versus temperature and oxygen versus sulfur partial pressures, respectively. From the relation of oxygen partial pressure versus temperature discussed by Barin and Platzki ${ }^{11)}$ and shown in Fig. 6(a), it is observed that $\mathrm{TiO}_{2}$ is reduced to metallic titanium via $\mathrm{Ti}_{4} \mathrm{O}_{7}$,

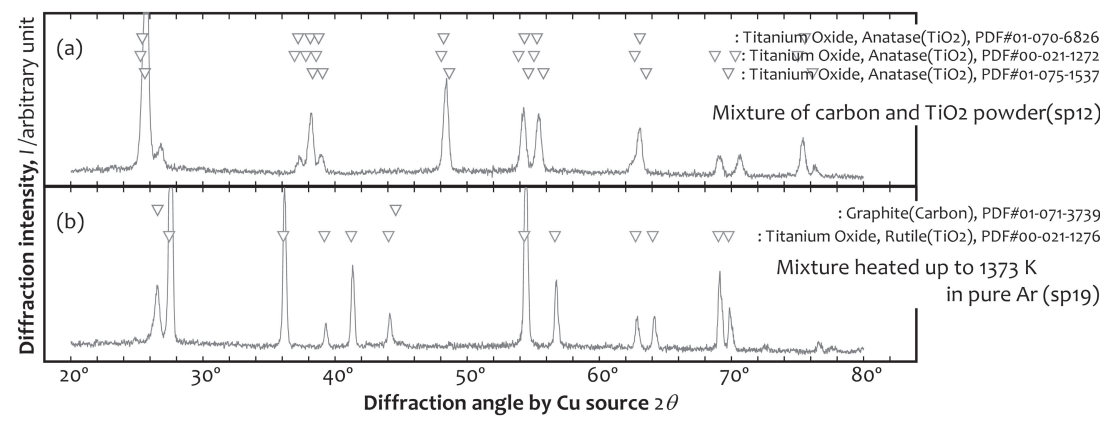

Fig. 4 X-ray diffraction patterns of (a) the mixture before the heat-treatment, and (b) that after the heat-treatment at $1373 \mathrm{~K}$ for $1.8 \mathrm{ks}$ in pure Ar atmosphere. 


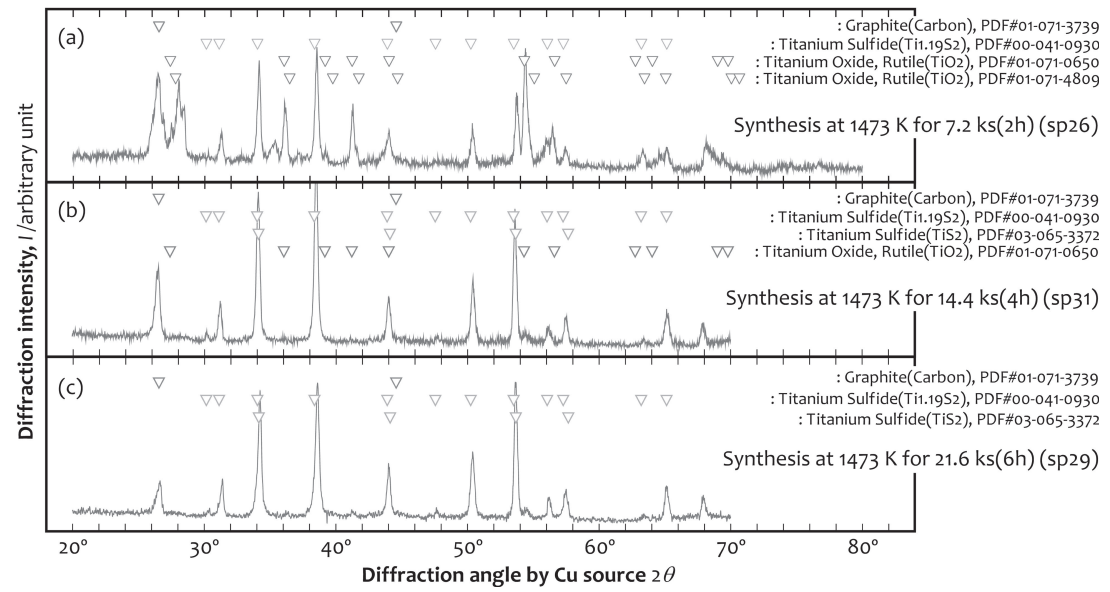

Fig. 5 X-ray diffraction patterns of the mixtures after the heat-treatments at $1473 \mathrm{~K}$ for (a) $7.2 \mathrm{ks}$, (b) $14.4 \mathrm{ks}$ and (c) $21.6 \mathrm{ks}$ with sulfurization.
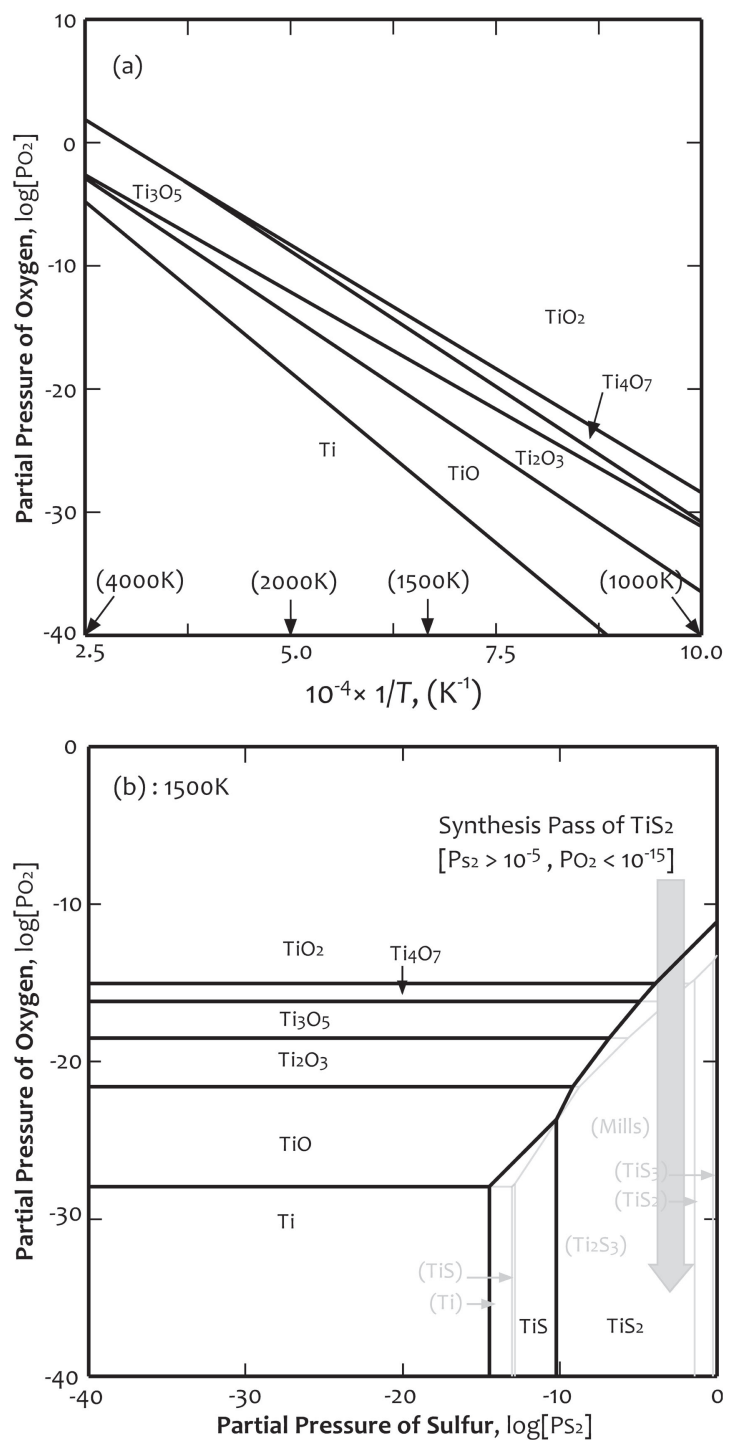

Fig. 6 Diagram of titanium for (a) oxygen partial pressure and temperature, and for (b) oxygen and sulfur partial pressures at $1500 \mathrm{~K}$, thermodynamically described by Barin ${ }^{11)}$ and Mills, ${ }^{20)}$ respectively.

$\mathrm{Ti}_{3} \mathrm{O}_{5}, \mathrm{Ti}_{2} \mathrm{O}_{3}$, and $\mathrm{TiO}$ in the wide temperature region including the synthesis temperature of $1473 \mathrm{~K}$. As observed from Fig. 6(b), when the synthesis temperature is fixed/set to
$1500 \mathrm{~K}$ (i.e., approximately $1473 \mathrm{~K}$ ), the titanium oxides are converted to the titanium sulfides with a decrease in oxygen partial pressures. The bold lines shown in the diagram represent the results discussed by Barin and Platzki, ${ }^{11)}$ and the weak (gray) lines represent those described by Mills. ${ }^{20)}$ Since the presence of other phases of titanium oxides, such as $\mathrm{Ti}_{4} \mathrm{O}_{7}, \mathrm{Ti}_{3} \mathrm{O}_{5}, \mathrm{Ti}_{2} \mathrm{O}_{3}$ and $\mathrm{TiO}$, are not confirmed from the XRD analysis, it is expected that $\mathrm{TiO}_{2}$ was directly converted to $\mathrm{TiS}_{2}$ as shown in Fig. 6(b). From the diagram, it is observed that $\mathrm{TiO}_{2}$ is directly converted to $\mathrm{TiS}_{2}$ under the sulfur partial pressure of more than $10^{-2} \mathrm{~atm}$. Barin and Platzki ${ }^{11)}$ confirm that the sulfur partial pressure becomes less than $10^{-5} \mathrm{~atm}$. Since the XRD results have shown the presence of $\mathrm{TiO}_{2}$ and $\mathrm{TiS}_{2}$, the thermodynamic relation reported by Barin and Platzki ${ }^{11)}$ can be used in the present study. Here, because the mixture coexists with the graphite powder, as mentioned in the experimental procedure part, the carbon potential is also interpreted to be $a_{\mathrm{c}}=1$ for the reaction. The equilibrium constant $(K)$ of the reaction, $2 \mathrm{CO} \rightarrow \mathrm{C}+\mathrm{CO}_{2}$, as obtained from the literature, ${ }^{11)}$ is given by $\log K=-3.18$ at $1500 \mathrm{~K}$. Thus, $\mathrm{CO}$ partial pressure at the situation is determined as $P_{\mathrm{CO}}=0.99 \mathrm{~atm}$. From the $\mathrm{CO} / \mathrm{CO}_{2}$ ratio, the oxygen partial pressure is also determined to be $P_{\mathrm{O} 2}=10^{-17} \mathrm{~atm}$, and for the reaction $\mathrm{CO}_{2} \rightarrow \mathrm{CO}+1 / 2 \mathrm{O}_{2}$, the value of equilibrium constant is $\log K=-5.31$ at $1500 \mathrm{~K}$. Since the determined oxygen partial pressure almost matches with that of the value obtained from the diagram shown in Fig. 6(b), we can confirm that $\mathrm{TiO}_{2}$ in the mixture is directly converted to $\mathrm{TiS}_{2}$ as depicted by the arrow of gray in the diagram.

\subsection{Thermal decomposition of titanium disulfide to produce metallic titanium}

Figure 7(a)-(f) illustrate the decomposition process of $\mathrm{TiS}_{2}$ to metallic titanium ingot. Figure 7(a) shows the initial state of the compressed $\mathrm{TiS}_{2}$ powder of sp29 converted for $21.6 \mathrm{ks}$, and Fig. 7(b) shows the reduced product exhibiting a lustrous surface. As the decomposition process progresses, rounded products with lustrous surfaces are formed depending on the heating time, as shown in Fig. 7(c)-(e). The sample shown by Fig. 7(a) reached the sample shown by Fig. 7(e) for few minutes except for purging time by argon 

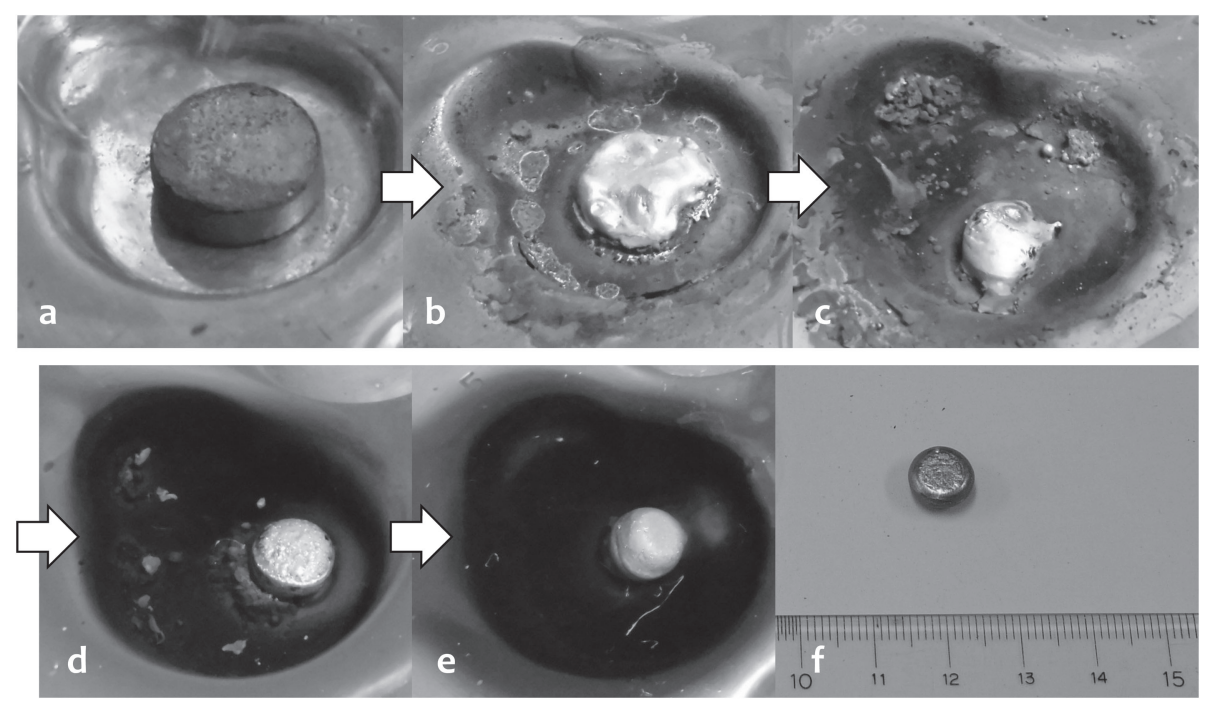

Fig. 7 Photographs of the reducing mixture by the thermal decomposition using the arc-flame of the arc melting furnace. (a) The compressed $\mathrm{TiS}_{2}$ prior to the reaction of sp29. (b)-(f) Partially thermal decomposed product having a lustrous surface, and renamed to be sp35.

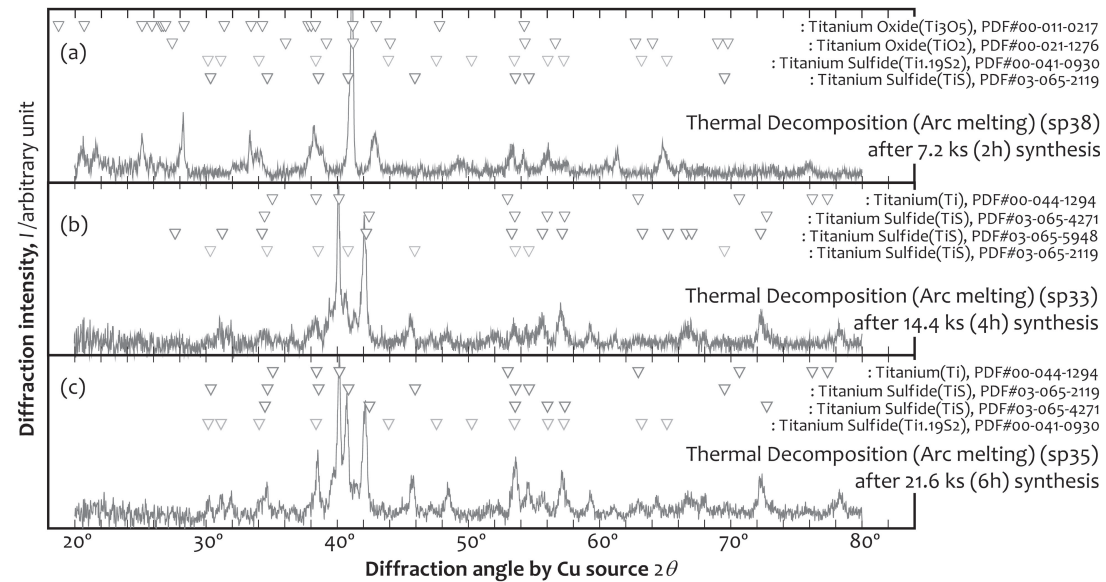

Fig. 8 X-ray diffraction patterns of the products after the thermal decomposition by the arc-flame for various synthesis conditions of (a) $7.2 \mathrm{ks}$, (b) $14.4 \mathrm{ks}$ and (c) $21.6 \mathrm{ks}$.

gas. The XRD patterns of the decomposed product shown in Fig. 7(f) re-named as sp35 and similarly prepared samples (sp26 and sp31 are re-named to be sp38 and sp33, respectively) are shown in Fig. 8(a)-(c). The XRD patterns of the arc-melted sample reacted for $7.2 \mathrm{ks}$ confirm the presence of titanium disulfide $\left(\mathrm{Ti}_{1.19} \mathrm{~S}_{2}\right.$, PDF \#00-041-0930) and titanium oxides $\left(\mathrm{TiO}_{2}, \mathrm{PDF} \# 00-021-1276\right.$ and $\mathrm{Ti}_{3} \mathrm{O}_{5}$, PDF \#00-011-0217) in the product, but there was no trace of metallic titanium in the mixture. However, as shown in Fig. 8(b)-(c), the mixtures reacted for 14.4 and $21.6 \mathrm{ks}$ confirm the presence of metallic titanium (Ti, PDF \#00-0441294) and titanium sulfide (TiS, PDF \#03-065-4271) with no trace of titanium oxides in those products. Figure 9(a)-(b) shows diagrams for sulfur partial pressure versus temperature and oxygen versus sulfur partial pressures, as reported by Barin and Platzki ${ }^{11)}$ and Mills, ${ }^{20)}$ respectively. Figure 9(a) shows the relation between sulfur partial pressure and temperature and suggests that $\mathrm{TiS}_{2}$ is directly reduced to metallic titanium above $2000 \mathrm{~K}$. During the reduction process, as the temperature in the arc furnace reaches
$4000 \mathrm{~K}$, the sulfur partial pressure decreases to $10^{-3} \mathrm{~atm}$. Since the sulfur partial pressure is decreased by purging an atmospheric pressure of argon gas, the reduction of $\mathrm{TiS}_{2}$ to metallic titanium is possible at this temperature. Hence, it can be interpreted that the XRD patterns should only contain the phases of metallic titanium. In contrast, since TiS is precipitated at a temperature less than $2000 \mathrm{~K}$, its presence in the $\mathrm{XRD}$ results can be interpreted as a precipitated phase during the cooling process after thermal decomposition. Additionally, the ingots synthesized for 14.4 and $21.6 \mathrm{ks}$ contained phases of reduced metallic titanium and TiS, whereas those synthesized for $7.2 \mathrm{ks}$ consisted phases of precipitated $\mathrm{Ti}_{3} \mathrm{O}_{5}$. Figure 9(b) suggest that the remaining $\mathrm{TiO}_{2}$ present in the synthesized mixture could not decrease the oxygen partial pressure sufficiently, indicating incomplete reduction of the mixture to metallic titanium. In other words, the synthesized mixture, which was completely reduced to $\mathrm{TiS}_{2}$, decreased the oxygen partial pressure to less than $10^{-5} \mathrm{~atm}$ as boundary of $\mathrm{Ti} / \mathrm{TiO}$, hence forming metallic titanium. Since, TiS is precipitated in the reduced ingot after 

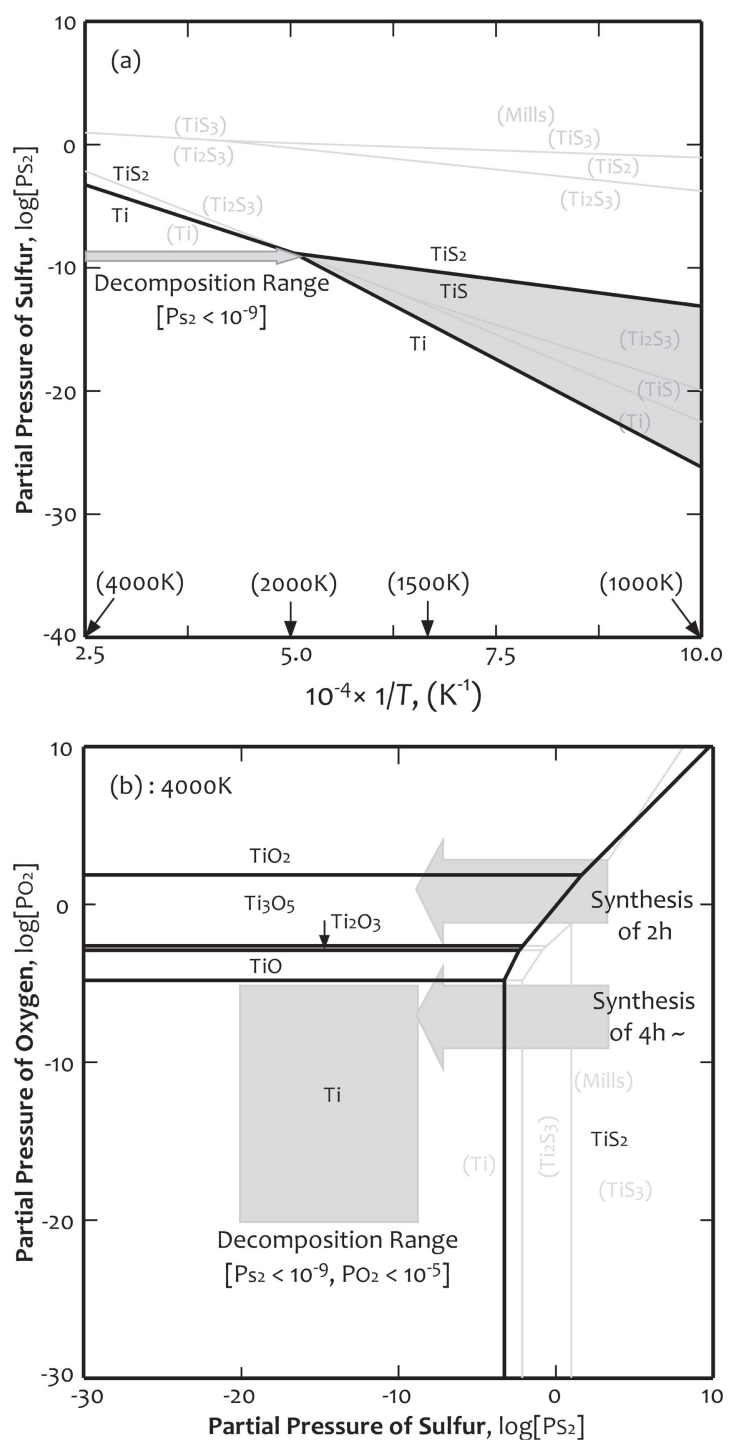

Fig. 9 Diagram of titanium for (a) sulfur partial pressure and temperature, and for (b) oxygen and sulfur partial pressures at $4000 \mathrm{~K}$ by Barin ${ }^{11)}$ and Mills, ${ }^{20)}$ respectively.

the thermal decomposition, the sulfur partial pressure is also interpreted to be less than $10^{-9} \mathrm{~atm}$ as limit of production of TiS at cooling during the experiments.

\section{Discussion}

Figure 10 shows a flowchart illustrating the conventional and proposed processing method for the manufacturing of metallic titanium ingot. The industrially performed conventional process uses an upgraded rutile, namely concentrated oxide as $\mathrm{TiO}_{2}$, and produces sponge-like metallic titanium via $\mathrm{TiCl}_{4}$ as the intermediate material, which is electrolytically reduced by metallic magnesium. Subsequently, the spongelike metallic titanium is subjected to vacuum distillation for the removal of $\mathrm{Mg}$ and $\mathrm{MgCl}_{2}$, and it forms titanium ingots by an arc melting. However, as both the magnesium and chlorine gas employed in the conventional process are electrochemically recycled, large amount of electricity is required for the electrolysis of $\mathrm{MgCl}_{2}$. Thus, the complex processing method and consumption of large amount of electricity increases the fabrication cost of metallic titanium. The above-mentioned constraints are overcome in the proposed technique, wherein $\mathrm{TiO}_{2}$ is reduced to metallic titanium ingots by thermolysis, with $\mathrm{TiS}_{2}$ used as the intermediate material, although the numerical comparison will be discussed in future. $\mathrm{TiS}_{2}$ is easily produced by the carbo-sulfurization under carbon potential-controlled sulfur gas atmosphere. Moreover, the reduction rate of $\mathrm{TiS}_{2}$ by the thermolysis does not depend on the surface properties, and seems to be fast. Hence, this method makes the fabrication of metallic titanium easy and cost-effective by eliminating certain complex steps, such as the electrochemical reduction of $\mathrm{MgCl}_{2}$ to produce metallic magnesium, vacuum distillation process, and compaction of the titanium sponge.

As mentioned earlier, the quality of produced titanium ingot is also maintained, since there is no trace of titanium oxides in the titanium ingot produced from the mixture heated for more than $14.4 \mathrm{ks}$. Although the solubility of oxygen in the metallic titanium is $33 \mathrm{at} \% \mathrm{O}(14 \mathrm{mass} \% \mathrm{O})^{16)}$ for wide temperature range, the oxygen content of the produced titanium ingot seems to be low, as the diffraction patterns of metallic titanium shown in Fig. 8(b), (c) matches well with the diffraction pattern of the standard metallic titanium, i.e., a remarkable effect causing with much solving impurity elements similar with titanium products reduced from $\mathrm{TiN}^{9}$ ) is not confirmed. Moreover, an arc-current of $150 \mathrm{~A}$ is used for the thermal decomposition of $\mathrm{TiS}_{2}$, which is significantly low than that used in case of TiN (250-300 A). $\left.{ }^{9}\right)$ As the reaction temperature is low when the arc-current is less, the chances of complete removal of impurities are low. The melting temperature of reduced titanium ingot is confirmed by the phase relations of $\mathrm{Ti}-\mathrm{S}^{16,21)}$ and $\mathrm{Ti}-\mathrm{N}$ systems. $^{22)}$ The binary system of Ti-S has a eutectic point, and the melting temperature of reduced titanium decreases on increasing the sulfur content, above the eutectic temperature, i.e., $1485 \mathrm{~K}$ for $14 \mathrm{at} \% \mathrm{~S}(10 \mathrm{mass} \% \mathrm{~S})$. In contrast, the binary system of $\mathrm{Ti}-\mathrm{N}$ has several peritectic points, and the melting temperature of titanium increases on increasing the nitrogen content above the peritectic temperature, i.e., $2323 \mathrm{~K}$ for beta-titanium of 9 at $\% \mathrm{~N}(2.8 \mathrm{mass} \% \mathrm{~N})$ and $2623 \mathrm{~K}$ for alphatitanium of 20 at $\% \mathrm{~N}(6.8$ mass $\% \mathrm{~N})$. The difference of the arc-current seems to be influenced by the phase-relation as one of reasons. The melting temperature of titanium for the binary system of $\mathrm{Ti}-\mathrm{O}$ increases depending on the oxygen content, which is $2158 \mathrm{~K}$ for 25 at $\% \mathrm{O}(10$ mass $\left.\% O) .{ }^{16}\right)$ Moreover, from the binary system of $\mathrm{Ti}-\mathrm{S}$, it is known that the sulfur is insoluble into the metallic titanium under the eutectic temperature. ${ }^{16,21)}$ The significant low solubility of sulfur is attributed to the precipitation of TiS in the titanium ingot produced by the thermal decomposition process. The partial pressure of sulfur of TiS precipitation is less than $10^{-9} \mathrm{~atm}$ under $2000 \mathrm{~K}$, as shown in Fig. 9(a). It may be possible to decrease the precipitation of TiS if the partial pressure of sulfur is further decreased (less than that in the present), with purging of atmosphere gas after the reaction in the reaction chamber by inert gas such as argon, but it is difficult to completely avoid the precipitation of TiS as it disperses as a eutectic structure. It may be possible to avoid precipitation and thus decrease the sulfur content from the 


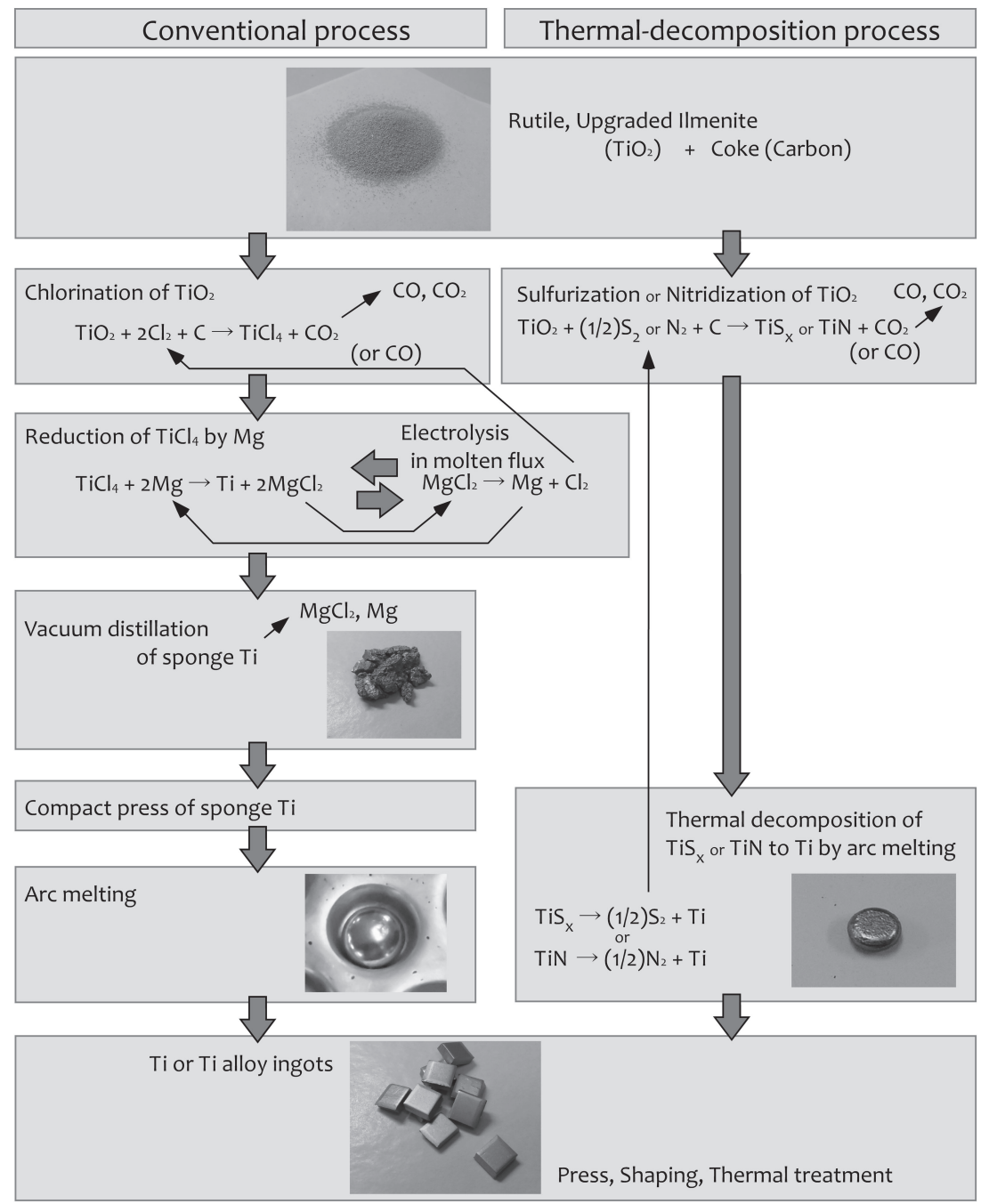

Fig. 10 Schematic flowchart of the commercial and presented processes of the metallic titanium ingot manufacturing.

reduced metallic titanium, through heat treatment, but it could not be achieved in the present study due to the limitations of the equipment. However, considering the characteristic phenomena of the Ti-S system, the treatment of $\mathrm{TiS}_{2}$ as the intermediate material for thermal decomposition process is favorable for the issues.

As mentioned earlier, the proposed method for the production of titanium ingot includes several impurity issues, but the results obtained indicate the separation of metallic titanium. However, extensive investigation and experiments, including quantitative investigation of the impurity elements are necessary for the clarification of the details of this approach via $\mathrm{TiS}_{2}$ as the intermediate material, and are oriented in further studies.

\section{Conclusions}

The proposed manufacturing process for reduction of $\mathrm{TiO}_{2}$ to metallic titanium by the thermal decomposition using $\mathrm{TiS}_{2}$ as the intermediate material was experimentally performed. The synthesized $\mathrm{TiS}_{2}$ was easily decomposed with a lower arc-current than that of previously reported TiN. Moreover, $\mathrm{TiS}_{2}$ was easily synthesized by the carbothermic reaction in sulfur atmosphere at a reasonable temperature above $1473 \mathrm{~K}$, which was lower than that of $\mathrm{TiN}$ in the previous study. The equipment for the synthesis of mixture, i.e., the conventional electric resistance furnace accompanied with sulfur gas generation system, was easily available. The subsequent decomposition of $\mathrm{TiS}_{2}$ was also performed in an electric arc furnace, and the produced metallic titanium had a lustrous surface. The phases of the synthesized mixture and the thermally decomposed titanium ingot were confirmed through XRD analysis, and were target phases.

\section{Acknowledgements}

This study was supported by the Wesco Scientific Promotion Foundation for the study of the fundamental synthesizing process of titanium sulfide, the Mukai Science and Technology Foundation for the production of high purity titanium sulfide for the novel manufacturing process of metallic titanium, and a cooperative program (202011CRKEQ-0003) of the Cooperative Research and Development Center for Advanced Materials, Institute for Materials Research (CTDAM-IMR), Tohoku University for the investigation of the decomposition process of titanium sulfide to metallic titanium. 


\section{REFERENCES}

1) W. Kroll: Trans. Electrochem. Soc. 78 (1940) 35-47.

2) R.O. Suzuki, S. Natsui and T. Kikuchi: Mater. Trans. 62 (2021) $905-$ 913.

3) G.Z. Chen, D.J. Fray and T.W. Farthing: Nature 407 (2000) 361-364

4) R.O. Suzuki, K. Teranuma and K. Ono: Metall. Mater. Trans. 34 (2003) 287-295.

5) R.O. Suzuki and S. Fukui: Mater. Trans. 45 (2004) 1665-1671.

6) I. Seki, K. Nagata, J. Tanabe, S. Yamaura and X. Wang: Mater. Trans. 55 (2014) 1419-1427.

7) O. Takeda and T.H. Okabe: Mater. Trans. 47 (2006) 1145-1154.

8) H. Zheng, H. Ito and T.H. Okabe: Mater. Trans. 48 (2007) 2244-2251.

9) I. Seki and S. Yamaura: Mater. Trans. 58 (2017) 361-366.

10) I. Seki: Mater. Trans. 58 (2017) 1546-1554.

11) I. Barin and G. Platzki: Thermochemical Data of Pure Substances, 3rd ed., (VCH Verlagsgesellschaft, Weinheim, 1995) pp. 1690-1696, 1701-1703.

12) N. Suzuki, M. Tanaka, H. Noguchi, S. Natsui, T. Kikuchi and R.O. Suzuki: ECS Trans. 75 (2016) 507-515.

13) N. Suzuki, M. Tanaka, H. Noguchi, S. Natsui, T. Kikuchi and R.O.
Suzuki: Mater. Trans. 58 (2017) 367-370.

14) M. Ohta, S. Satoh, T. Kuzuya, S. Hirai, M. Kunii and A. Yamamoto: Acta Mater. 60 (2012) 7232-7240.

15) E. Ahmadi, Y. Yashima, R.O. Suzuki and S.A. Rezan: Metall. Mater. Trans. B 49 (2018) 1808-1821.

16) J.L. Muray: Phase Diagram of Binary Titanium Alloys, Monograph Series on Alloy Phase Diagrams, (ASM International, Metals Park, Ohio, 1987) pp. 211-229, 275-282.

17) K. Kubo and K. Shinriki: Kogyō Kagaku Zassi (J. Soc. Chem. Ind., Japan) 56 (1953) 149-151.

18) S. Takeuchi and H. Katsuta: J. Japan Inst. Met. Mater. 34 (1970) 758763.

19) S. Motojima, K. Itoh, Y. Takahashi and K. Sugiyama: Bull. Chem. Soc. Jpn. 51 (1978) 3240-3244.

20) K.C. Mills: Thermodynamic Data for Inorganic Sulphides, Selenides and Tellurides, (Butterworth \& Co. Publishers Ltd., London, 1974) pp. 56-57.

21) K. Hirata, S. Iikubo, H. Fujimoto and H. Ohtani: Calphad 57 (2017) 62-77.

22) C. Vahlas, B.D. Ladouce, P.Y. Chevalier, C. Bernard and L. Vanden Bulcke: Thermochim. Acta 180 (1991) 23-37. 